Теорія Ймовір. та Матем. Статист. Вип. 76, 2007
Theor. Probability and Math. Statist.

No. 76, 2008, Pages 181-197

S 0094-9000(08)00742-4

Article electronically published on July 17, 2008

\title{
CONSISTENCY OF AN ESTIMATOR OF THE PARAMETERS OF A POLYNOMIAL REGRESSION WITH A KNOWN VARIANCE RELATION FOR ERRORS IN THE MEASUREMENT OF THE REGRESSOR AND THE ECHO
}

UDC 519.21

\author{
S. V. SHKLYAR
}

\begin{abstract}
We consider an error-in-variables model for a polynomial regression with Gaussian errors. We assume that the covariance matrix of the measurement errors of the regressor and the echo is known up to a scalar factor. We consider the moment estimator of regression coefficients proposed by Cheng and Schneeweiss. Sufficient conditions for the strong consistency of this estimator are given and the rate of convergence is estimated in this paper.
\end{abstract}

\section{INTRODUCTION}

We consider a functional model of a polynomial regression with Gaussian measurement errors. This model is studied in [1.

Cheng and Schneeweiss [2, 3] proposed an estimator for the regression parameters for the case where the covariance matrix of measurement errors is known up to a scalar factor. The estimator is constructed by using the method of moments, in fact. However the conditions for the consistency of this estimator are not given in [2, 3.

Let $\left\{\xi_{i}, i=1,2, \ldots\right\}$ be a sequence of nonrandom numbers,

$$
\eta_{i}=\bar{\beta}_{0}+\bar{\beta}_{1} \xi_{i}+\bar{\beta}_{2} \xi_{i}^{2}+\cdots+\bar{\beta}_{k} \xi_{i}^{k}, \quad i=1,2, \ldots
$$

Here $\bar{\beta}=\left(\bar{\beta}_{0}, \bar{\beta}_{1}, \ldots, \bar{\beta}_{k}\right)^{\top}$ is a parameter and $k \geq 1$ is a fixed known number. Assume that the pairs $\left(\xi_{i}, \eta_{i}\right)$ are observed with an error, that is, the pairs $\left(x_{i}, y_{i}\right), i=1, \ldots, n$, are observed where

$$
x_{i}=\xi_{i}+\delta_{i}, \quad y_{i}=\eta_{i}+\varepsilon_{i} .
$$

The measurement errors are independent and have the same Gaussian distribution:

$$
\left(\delta_{i}, \varepsilon_{i}\right)^{\top} \sim N\left(0, \bar{\kappa} \Omega_{0}\right) .
$$

The covariance matrix $\bar{\kappa} \Omega_{0}$ is known up to the factor $\bar{\kappa} \geq 0$. The matrix

$$
\Omega_{0}=\operatorname{diag}\left(\sigma_{\delta 0}^{2}, \sigma_{\varepsilon 0}^{2}\right)
$$

is diagonal and $\sigma_{\varepsilon 0}>0$ and $\sigma_{\delta 0}>0$ are known.

The problem is to estimate the parameter $\bar{\beta}$ from the observations.

Below we list some known estimators. First we mention some "naive" estimation methods for the error-in-variables models. These methods neglect measurement errors for the regressor.

2000 Mathematics Subject Classification. Primary 62J02; Secondary 62F10, 62F12, 62J10.

Key words and phrases. Polynomial regression, error-in-variables model. 
Consistent estimators that do not use any additional assumption on the parameters are discussed in the book [1. Consistent estimators are considered in the paper [3] for the case where $\sigma_{\delta}^{2}=\bar{\kappa} \sigma_{\delta 0}^{2}$ is known. Among others, these estimators include MALS, WLS (used if both variances $\sigma_{\delta}^{2}$ and $\sigma_{\varepsilon}^{2}$ are known), and SLS (for a structural model where the regressor has a Gaussian distribution).

There are some inconsistent estimators used for the case where the ratio $\sigma_{\delta}^{2} / \sigma_{\varepsilon}^{2}$ is known. Among those estimators are the orthogonal regression estimator and its generalization [4] and an adjusted Kanatani estimator [5].

In addition to the Cheng-Schneeweiss estimator one can construct some other estimators if the ratio $\sigma_{\delta}^{2} / \sigma_{\varepsilon}^{2}$ is known (one of the approaches could be to consider terms with weights in the estimation function similarly to [5, $\S \S 6-7]$ ); note however that results of this kind are unknown to the author.

Kukush et al. [6] propose a consistent estimator for an implicit second order model. They consider both cases if the covariance matrix of errors is known and if this matrix is proportional to the unit matrix with unknown proportion coefficient. Shklyar et al. [7] improved the conditions for the consistency of the estimator in the second case.

In this paper, we prove the consistency of the Cheng-Schneeweiss estimator under conditions similar to those in [7]. The standard theorem on the consistency of the moment estimator cannot be used in the case under consideration, since the observations are nonidentically distributed. The estimator for the structural model uses sampling moments that can correspond to infinite theoretical moments.

We are mainly concerned with the functional model. The estimator is defined in Section 2. Conditions for the consistency of the functional and structural models are stated in Section 3. The proof is given in Section 5 for the functional model and in Section 7 for the structural model. Section [6] contains a discussion on possible improvements of the conditions. In Section 4 we consider some transformations of the equality for the estimator.

\section{The Estimator}

Let

$$
\psi(\xi, \eta)=\left(\eta, 1, \xi, \xi^{2}, \ldots, \xi^{k}\right)^{\top}\left(\eta, 1, \xi, \xi^{2}, \ldots, \xi^{k}\right) .
$$

Denote by $\psi(x, y, \kappa)$ a solution of the deconvolution problem; namely, $\psi(x, y, \kappa)$ is a symmetric matrix whose entries are polynomials in $x$ and $y$ such that

$$
\mathrm{E} \psi(x, y, \kappa)=\psi(\xi, \eta), \quad\left(\begin{array}{l}
x \\
y
\end{array}\right) \sim N\left(\left(\begin{array}{l}
\xi \\
\eta
\end{array}\right), \kappa \Omega_{0}\right)
$$

for all $\xi, \eta \in \mathbb{R}$. The coefficients of the polynomials may depend on $\kappa \Omega_{0}$. The first entries of the matrix $\psi(x, y, \kappa)$ are as follows:

$$
\begin{aligned}
& \psi(x, y, \kappa) \\
& =\left(\begin{array}{ccccc}
y^{2}-\kappa \sigma_{\varepsilon 0}^{2} & y & x y & x^{2} y-y \kappa \sigma_{\delta 0}^{2} & \ldots \\
y & 1 & x & x^{2}-\kappa \sigma_{\delta 0}^{2} & \ldots \\
x y & x & x^{2}-\kappa \sigma_{\delta 0}^{2} & x^{3}-3 x \kappa \sigma_{\delta 0}^{2} & \ldots \\
x^{2} y-y \kappa \sigma_{\delta 0}^{2} & x^{2}-\kappa \sigma_{\delta 0}^{2} & x^{3}-3 x \kappa \sigma_{\delta 0}^{2} & x^{4}-6 x^{2} \kappa \sigma_{\delta 0}^{2}+3 \kappa^{2} \sigma_{\delta 0}^{4} & \ldots \\
\ldots \ldots \ldots \ldots \ldots \ldots \ldots \ldots \ldots \ldots \ldots \ldots \ldots \ldots \ldots \ldots \ldots \ldots \ldots \ldots \ldots \ldots \ldots \ldots \ldots \ldots \ldots \ldots \ldots \ldots \ldots \ldots \ldots \ldots \ldots \ldots \ldots
\end{array}\right) .
\end{aligned}
$$

Relation (10) and formulas (7) and (10) below determine explicitly the matrix for $k=2$. Now we can write the entries of the matrix $\psi(x, y, \kappa)=\left(\psi_{i j}\right)_{i, j=-1}^{k}$. We have

$$
\begin{gathered}
\psi_{-1,-1}=y^{2}-\kappa \sigma_{\varepsilon 0}^{2}, \quad \psi_{-1, i}=\psi_{i,-1}=y t_{i}(x), \quad i=0, \ldots, k, \\
\psi_{i j}=t_{i+j}(x), \quad i, j=0, \ldots, k,
\end{gathered}
$$


where

$$
t_{i}(x)=\left(\frac{1}{2} \kappa \sigma_{\delta 0}^{2}\right)^{i / 2} H\left(\frac{x}{\sqrt{2 \kappa} \sigma_{\delta 0}}\right)
$$

is the scaled Hermite polynomial with unit leading coefficient. The blocks constituting the matrix $\psi(x, y, \kappa)$ are defined in the paper [3]. According to the notation of $[3]$,

$$
\psi\left(x_{i}, y_{i}, \kappa\right)=\left(\begin{array}{cc}
y_{i}^{2}-\sigma_{\varepsilon}^{2} & \boldsymbol{h}_{i} \\
\boldsymbol{h}_{i}^{\top} & \boldsymbol{H}_{i}
\end{array}\right) .
$$

Put

$$
\bar{\Psi}=\sum_{i=1}^{n} \psi\left(\xi_{i}, \eta_{i}\right), \quad \Psi(\kappa)=\sum_{i=1}^{n} \psi\left(x_{i}, y_{i}, \kappa\right) .
$$

The matrices $\bar{\Psi}$ and $\Psi(\kappa)$ depend on the size $n$ of the sample. We have $\mathrm{E} \Psi(\bar{\kappa})=\bar{\Psi}$.

The estimator of the parameters $\bar{\beta}$ and $\bar{\kappa}$ is defined as a solution of the equation

$$
\Psi(\hat{\kappa})\left(\begin{array}{c}
-1 \\
\hat{\beta}
\end{array}\right)=0 .
$$

Equation (2) may have several solutions. In those cases, one can use one of the following two approaches when choosing the estimator $\hat{\kappa}$ for the parameter $\bar{\kappa}$ :

- choose the minimal $\kappa \geq 0$ such that the matrix $\Psi(\kappa)$ is degenerate;

- choose $\kappa \geq 0$ such that the matrix $\Psi(\kappa)$ is degenerate and nonnegative definite, that is, $\lambda_{\min }(\Psi(\kappa))=0$ where $\lambda_{\min }(\Psi)$ is the minimal eigenvalue of the symmetric matrix $\Psi$.

Both approaches above are equivalent in view of the following result.

Theorem 2.1. The equation $\lambda_{\min }(\Psi(\kappa))=0$ has a unique solution $\kappa \geq 0$ denoted by $\hat{\kappa}$. If $0 \leq \kappa<\hat{\kappa}$, then the matrix $\Psi(\kappa)$ is positive definite. If $\kappa>\hat{\kappa}$, then the matrix $\Psi(\kappa)$ is not definite.

Here and in what follows we say that a real symmetric matrix $\Psi$ is not definite if

$$
\lambda_{\min }(\Psi)<0<\lambda_{\max }(\Psi) .
$$

Theorem 2.1 is proved in Section 4.3 .

Following one of the above approaches we choose $\hat{\kappa}$ and then evaluate $\hat{\beta}$ from equation (2). If $\Psi(\hat{\kappa})\left(\begin{array}{l}0 \\ \beta\end{array}\right)=0$ for some $\beta \in \mathbb{R}^{k} \backslash\{0\}$, then the estimator is not defined.

\section{MAIN RESUlts}

3.1. Main results for the functional model. Here we state the results on the consistency and rate of convergence of the estimator. The proofs of these results are given at the end of Section 5 .

Theorem 3.1. Let

(i) there exist a number $C_{1}>0$ such that

$$
\lambda_{\min }\left(\frac{1}{n} \sum_{i=1}^{n}\left(\begin{array}{c}
1 \\
\xi_{i} \\
\vdots \\
\xi_{i}^{k}
\end{array}\right)\left(\begin{array}{c}
1 \\
\xi_{i} \\
\vdots \\
\xi_{i}^{k}
\end{array}\right)^{\top}\right) \geq \frac{1}{C_{1}}
$$

for sufficiently large $n$;

(ii) $\sum_{n=1}^{\infty} \xi_{n}^{4 k-2} / n^{2}<\infty$;

(iii) $\beta_{k} \neq 0$, that is, the polynomial used to express the dependence of $\eta_{i}$ on $\xi_{i}$ is of degree $k$. 
Then

$$
\hat{\beta} \stackrel{\mathrm{P} 1}{\longrightarrow} \bar{\beta}, \quad n \rightarrow \infty
$$

and

$$
\hat{\kappa} \stackrel{\mathrm{P} 1}{\longrightarrow} \bar{\kappa}, \quad n \rightarrow \infty .
$$

For the proof of Theorem 3.1 we use the convergence

$$
\frac{1}{n}\|\Psi(\bar{\kappa})-\bar{\Psi}\| \stackrel{\mathrm{P} 1}{\longrightarrow} 0, \quad n \rightarrow \infty
$$

(see Lemma 5.1 below). The symbol " $\stackrel{\mathrm{P} 1}{\longrightarrow}$ ” stands for the almost sure convergence.

Now we state the result about the rate of convergence.

Theorem 3.2. Assume that conditions (i), (ii), and (iii) hold. Then

$$
\begin{array}{ll}
\hat{\beta}-\bar{\beta}=O\left(\frac{1}{n}\|\Psi(\bar{\kappa})-\bar{\Psi}\|\right), & n \rightarrow \infty, \\
\hat{\kappa}-\bar{\kappa}=O\left(\frac{1}{n}\|\Psi(\bar{\kappa})-\bar{\Psi}\|\right), & n \rightarrow \infty,
\end{array}
$$

almost surely.

3.2. Consistency for the structural model. We prove the consistency for the structural model by using the corresponding proof for the functional model. Below we assume that $\xi_{i}, i=1,2, \ldots$, are independent identically distributed random variables that are independent of the measurement errors.

Theorem 3.3. Assume that

(Si) for all sets $\Xi \subset \mathbb{R}$ consisting of $k$ points,

$$
\mathrm{P}\left(\xi_{1} \in \Xi\right)<1
$$

(Sii) $\mathrm{E}\left|\xi_{1}\right|^{2 k-1}<\infty$;

(iii) $\bar{\beta}_{k} \neq 0$.

Then $\hat{\kappa}$ and $\hat{\beta}$ are consistent estimators of the parameters $\bar{\kappa}$ and $\bar{\beta}$, respectively.

The proof of this result is given in Section 7

\section{Change of parameters}

In this section, we establish an equation for the estimator of regression parameters in a form useful for the proof of the consistency.

\subsection{An equality for $\Psi(\kappa)$.}

Lemma 4.1. Let a vector $(X, Y)^{\top}$ have a Gaussian distribution in $\mathbb{R}^{2}$. Then

$$
\mathrm{E} X^{l} \mathrm{E} Y^{m}=\sum_{j=0}^{\min \{l, m\}} \frac{l ! m ! \mathrm{E} X^{l-j} Y^{m-j}(-\operatorname{cov}(X, Y))^{j}}{j !(l-j) !(m-j) !} .
$$

Proof. 1. First we consider the case of $X=Y \sim N(0,1)$. Then the moment $\mathrm{E} X^{k}$ equals the total number of ways to split a set of $k$ elements into $k / 2$ pairs (if $k$ is odd, let this number be 0 ). Consider a set consisting of $l$ elements of the first kind and $m$ elements of the second kind. Then

$$
\frac{l ! m !}{j !(l-j) !(m-j) !}
$$


equals the total number of ways to form $j$ disjoint pairs whose elements are of different kinds. The moment $\mathrm{E} X^{l-j} Y^{m-j}$ equals the total number of ways to complete those $j$ pairs up to $\frac{1}{2}(l+m)$ pairs. Note that $-\operatorname{cov}(X, Y)=-1$ and $\mathrm{E} X^{l} \mathrm{E} Y^{m}$ equals the total number of ways to form $\frac{1}{2}(l+m)$ pairs whose elements are of the same kind. Thus equality (5) follows from the inclusion-exclusion principle.

2. Let equality (5) hold for a certain distribution of the random variables $X$ and $Y$. Then

$$
\mathrm{E}(a X)^{l} \mathrm{E}(b Y)^{m}=\sum_{j=0}^{\min \{l, m\}} \frac{l ! m ! \mathrm{E}(a X)^{l-j}(b Y)^{m-j}(-\operatorname{cov}(a X, b Y))^{j}}{j !(l-j) !(m-j) !} .
$$

Thus equality (5) also holds for the pair of random variables $a X$ and $b Y$.

3. Assume that a random variable $Z$ does not depend on $(X, Y)$ and $\mathrm{E}|Z|^{l}<\infty$. We prove that if equality (5) holds for $X$ and $Y$, then it also holds for $X+Z$ and $Y$. Indeed,

$$
\begin{aligned}
\mathrm{E}(X+Z)^{l} \mathrm{E} Y^{m} & =\sum_{t=0}^{l} C_{l}^{t} \mathrm{E} Z^{t} \mathrm{E} X^{l-t} \mathrm{E} Y^{m} \\
& =\sum_{(t, j) \in M} \frac{l ! m ! \mathrm{E} Z^{t} \mathrm{E}\left[X^{l-t-j} Y^{m-j}\right](-\operatorname{cov}(X, Y))^{j}}{t ! j !(l-t-j) !(m-j) !} \\
& =\sum_{j=0}^{\min \{l, m\}} \frac{l ! m !}{j !(l-j) !(m-j) !} \sum_{t=0}^{l-j} C_{l-j}^{t} \mathrm{E}\left[Z^{t} X^{l-t-j} Y^{m-j}\right](-\operatorname{cov}(X, Y))^{j} \\
& =\sum_{j=0}^{\min \{l, m\}} \frac{l ! m ! \mathrm{E}\left[(X+Z)^{l-j} Y^{m-j}\right](-\operatorname{cov}(X+Z, Y))^{j}}{j !(l-j) !(m-j) !},
\end{aligned}
$$

where

$$
M=\left\{(t, j) \in(\mathbb{N} \cup\{0\})^{2} \mid t+j \leq l, j \leq m\right\} .
$$

Similarly, equality (5) holds for $X$ and $Y+Z$ if we assume that $\mathrm{E}|Z|^{m}<\infty$ instead of $\mathrm{E}|Z|^{l}<\infty$.

4. Using the above two steps we prove equality (5) step by step for the following distributions of the vector $(X, Y)^{\top}$ :

$$
\begin{gathered}
N\left(\left(\begin{array}{l}
0 \\
0
\end{array}\right),\left(\begin{array}{ll}
1 & 1 \\
1 & 1
\end{array}\right)\right), \quad N\left(\left(\begin{array}{l}
0 \\
0
\end{array}\right),\left(\begin{array}{cc}
\sigma_{X Y}^{2} / \sigma_{Y}^{2} & \sigma_{X Y} \\
\sigma_{X Y} & \sigma_{Y}^{2}
\end{array}\right)\right), \\
N\left(\left(\begin{array}{c}
\mu_{X} \\
0
\end{array}\right),\left(\begin{array}{cc}
\sigma_{X}^{2} & \sigma_{X Y} \\
\sigma_{X Y} & \sigma_{Y}^{2}
\end{array}\right)\right), \quad N\left(\left(\begin{array}{c}
\mu_{X} \\
\mu_{Y}
\end{array}\right),\left(\begin{array}{cc}
\sigma_{X}^{2} & \sigma_{X Y} \\
\sigma_{X Y} & \sigma_{Y}^{2}
\end{array}\right)\right),
\end{gathered}
$$

where $\left(\begin{array}{cc}\sigma_{X}^{2} & \sigma_{X Y} \\ \sigma_{X Y} & \sigma_{Y}^{2}\end{array}\right)$ is an arbitrary nonnegative definite matrix such that $\sigma_{Y}^{2} \neq 0$. The case of $\sigma_{Y}^{2}=0$ is trivial.

Consider the following $(k+2) \times(k+2)$ matrices:

$$
D_{x}=\left(\begin{array}{cccccc}
0 & 0 & 0 & \ldots & 0 & 0 \\
0 & 0 & 0 & & 0 & 0 \\
0 & 1 & 0 & & 0 & 0 \\
0 & 0 & 2 & \ddots & 0 & 0 \\
\ldots & \ldots & \ldots & \ldots & \ldots & \ldots \\
0 & 0 & 0 & \ldots & k & 0
\end{array}\right), \quad D_{y}=\left(\begin{array}{ccc}
0 & 1 & \\
0 & 0 & 0_{2 \times k} \\
0_{k \times 2} & 0_{k \times k}
\end{array}\right)
$$

If $\Psi$ is a $(k+2) \times(k+2)$ matrix, then we put

$$
R(\Psi)=\sigma_{\delta 0}^{2} D_{x} \Psi D_{x}^{\top}+\sigma_{\varepsilon 0}^{2} D_{y} \Psi D_{y}^{\top} .
$$


The operator $R$ preserves the nonnegative definiteness, namely if $\Psi \geq 0$, then $R(\Psi) \geq 0$. Moreover

$$
\begin{gathered}
R^{j}(\Psi)=\sigma_{\delta 0}^{2 j} D_{x}^{j} \Psi D_{x}^{j \top}, \quad j \geq 2, \\
R^{j}(\Psi)=0, \quad j>k .
\end{gathered}
$$

Thus $R$ is a nilpotent operator.

By $K(\kappa)$ we denote the $(k+2) \times(k+2)$ matrix such that

$$
\mathrm{E} K(\kappa)\left(y, 1, x, x^{2}, \ldots, x^{k}\right)^{\top}=\left(\eta, 1, \xi, \xi^{2}, \ldots, x^{k}\right)^{\top}, \quad\left(\begin{array}{l}
x \\
y
\end{array}\right) \sim N\left(\left(\begin{array}{l}
\xi \\
\eta
\end{array}\right), \kappa \Omega_{0}\right) .
$$

Note that all entries of the matrix $K(\kappa)$ above the principal diagonal are zero and its diagonal entries are equal to one. Moreover $K(0)$ is the unit matrix.

The first entries of the matrix $K(\kappa)$ are given by

$$
K(\kappa)=\left(\begin{array}{ccccc}
1 & 0 & 0 & 0 & \ldots \\
0 & 1 & 0 & 0 & \ldots \\
0 & 0 & 1 & 0 & \ldots \\
0 & -\kappa \sigma_{\delta 0}^{2} & 0 & 1 & \ldots \\
\ldots & \ldots & \ldots & \ldots
\end{array}\right)
$$

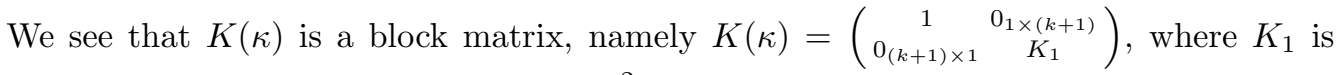
defined in 8 for the case of $\delta \sim N\left(0, \kappa \sigma_{\varepsilon 0}^{2}\right)$.

According to Lemma 4.1,

$$
\mathrm{E}\left(\begin{array}{c}
y \\
1 \\
x \\
\vdots \\
x^{k}
\end{array}\right) \mathrm{E}\left(\begin{array}{c}
y \\
1 \\
x \\
\vdots \\
x^{k}
\end{array}\right)^{\top}=\sum_{j=0}^{k} \frac{(-\kappa)^{j}}{j !} R^{j}(\mathrm{E} \psi(x, y))
$$

This implies that

$$
\psi(\xi, \eta)=\mathrm{E} K(\kappa)\left(\begin{array}{c}
y \\
1 \\
x \\
\vdots \\
x^{k}
\end{array}\right) \mathrm{E}\left(\begin{array}{c}
y \\
1 \\
x \\
\vdots \\
x^{k}
\end{array}\right)^{\top} K(\kappa)^{\top}=K(\kappa) \sum_{j=0}^{k} \frac{(-\kappa)^{j}}{j !} R^{j}(\mathrm{E} \psi(x, y)) K(\kappa)^{\top} .
$$

Thus

$$
\begin{gathered}
\psi(x, y, \kappa)=K(\kappa) \sum_{j=0}^{k} \frac{(-\kappa)^{j}}{j !} R^{j}(\psi(x, y)) K(\kappa)^{\top}, \\
\Psi(\kappa)=K(\kappa) \sum_{j=0}^{k} \frac{(-\kappa)^{j}}{j !} R^{j}(\Psi(0)) K(\kappa)^{\top} .
\end{gathered}
$$

4.2. Notation. Let

$$
\begin{gathered}
\Psi_{0}(\kappa)=K(\kappa)^{-1} \Psi(\kappa) K(\kappa)^{-\top} \\
\Psi_{j}(\kappa)=R^{j}\left(\Psi_{0}(\kappa)\right) .
\end{gathered}
$$


Equality (8) implies for $\kappa \geq 0$ that $\Psi_{0}(\kappa)=e^{-\kappa R}(\Psi(0))$, where $e^{-\kappa R}$ is the exponent of the nilpotent operator $-\kappa R$. Hence

$$
\Psi_{0}\left(\kappa_{2}\right)=e^{\left(\kappa_{1}-\kappa_{2}\right) R} \Psi_{0}\left(\kappa_{1}\right)=\sum_{j=0}^{k} \frac{\left(\kappa_{1}-\kappa_{2}\right)^{j}}{j !} \Psi_{j}\left(\kappa_{1}\right)
$$

for all $\kappa_{1} \geq 0$ and $\kappa_{2} \geq 0$.

Put $\bar{\Psi}_{0}=K(\bar{\kappa})^{-1} \overline{\bar{\Psi}} K(\bar{\kappa})^{-\top}$ and $\bar{\Psi}_{j}=R^{j}\left(\bar{\Psi}_{0}\right)$. The matrices $\bar{\Psi}_{j}$ are nonnegative definite for $j=0,1, \ldots$, since the matrix $\bar{\Psi}$ is nonnegative definite.

Let $\tilde{\psi}_{1}(\xi)=R\left(K(\bar{\kappa})^{-1} \psi(\xi, \eta) K(\bar{\kappa})^{-\top}\right)$. Note that the latter expression does not depend on $\eta$ and moreover $\tilde{\psi}_{1}(\xi)$ is a nonnegative definite matrix whose right-bottom entry is a polynomial of degree $2 k-2$ considered at the argument $\xi$, while all other entries are polynomials of smaller degrees. Thus $\bar{\Psi}_{1}=\sum_{i=1}^{n} \tilde{\psi}_{1}\left(\xi_{i}\right)$.

\subsection{An estimator of the parameter $\bar{\kappa}$.}

Lemma 4.2. If $0 \leq \kappa_{1}<\kappa_{2}$ and the matrix $\Psi\left(\kappa_{2}\right)$ is nonnegative definite, then $\Psi\left(\kappa_{1}\right)$ is positive definite.

Proof. Consider a matrix $\mathrm{Xl}(\delta, \varepsilon)$ such that

$$
\mathrm{Xl}(\delta, \varepsilon)\left(\eta, 1, \xi, \xi^{2}, \ldots, \xi^{k}\right)^{\top}=\left(\eta+\varepsilon, 1, \xi+\delta,(\xi+\delta)^{2}, \ldots,(\xi+\delta)^{k}\right)^{\top} .
$$

The matrix $\operatorname{Xl}(\delta, \varepsilon)$ is nondegenerate, since $\operatorname{det} \mathrm{Xl}(\delta, \varepsilon)=1$, its diagonal entries are units, and

$$
\mathrm{Xl}(\delta, \varepsilon)=\left(\begin{array}{ccccccc}
1 & \varepsilon & 0 & \ldots & 0 & \ldots & 0 \\
0 & 1 & 0 & \ldots & 0 & \ldots & 0 \\
0 & \delta & 1 & \ldots & 0 & \ldots & 0 \\
\ldots & \ldots & \ldots & \ldots \ldots & \ldots \ldots & \ldots & \ldots \\
0 & \delta^{k} & k \delta^{k-1} & \ldots & C_{k}^{j} \delta^{k-j} & \ldots & 1
\end{array}\right)
$$

where $C_{k}^{j}$ denotes the binomial coefficient $\left(\begin{array}{c}k \\ j\end{array}\right)$. Formally,

$$
\mathrm{Xl}(\varepsilon, \delta)=\left(\mathrm{xl}_{i j}\right)_{i, j=-1}^{k},
$$

where $\mathrm{xl}_{-1,-1}=1, \mathrm{xl}_{-1,0}=\varepsilon$, and

$$
\mathrm{xl}_{i j}=C_{i}^{j} \delta^{i-j}
$$

for $i \geq j \geq 0$, while all the other entries of the matrix are equal to 0 . Note that the entry $\mathrm{xl}_{i 0}$ is a monomial of a highest degree among the entries

$$
\left\{\mathrm{xl}_{i j},-1 \leq j \leq k\right\}
$$

of the $i$ th row.

We have

$$
\mathrm{Xl}(\delta, \varepsilon) \psi(\xi, \eta) \mathrm{Xl}(\delta, \varepsilon)^{\top}=\psi(\xi+\delta, \eta+\varepsilon) .
$$

One can check that

$$
\psi\left(x, y, \kappa_{1}\right)=\mathrm{E} \psi\left(x+\delta, y+\varepsilon, \kappa_{2}\right) .
$$

Here and in what follows, $\left(\begin{array}{l}\delta \\ \varepsilon\end{array}\right) \sim N\left(0,\left(\kappa_{2}-\kappa_{1}\right) \Omega_{0}\right)$. Thus

$$
\Psi\left(\kappa_{1}\right)=\operatorname{EXl}(\delta, \varepsilon) \Psi\left(\kappa_{2}\right) \operatorname{Xl}(\delta, \varepsilon)^{\top} .
$$

Consider the polynomial

$$
P(\delta, \varepsilon)=\theta^{\top} \mathrm{Xl}(\delta, \varepsilon) \Psi(\kappa) \mathrm{Xl}(\delta, \varepsilon)^{\top} \theta
$$


for $\theta^{\top}:=\left(\theta_{-1}, \theta_{0}, \theta_{1}, \ldots, \theta_{k}\right)^{\top} \neq 0$. Then

$$
P(\delta, \varepsilon) \geq 0
$$

for all $(\delta, \varepsilon)$. The polynomial $P(\delta, \varepsilon)$ contains a monomial $n \theta_{-1}^{2} \varepsilon^{2}$ if $\theta_{-1} \neq 0$. If $\theta_{k^{*}} \neq 0$ for some $k^{*}, 1 \leq k^{*} \leq k$, and $\theta_{j}=0$ for all $k^{*}<j \leq k$, then the polynomial $P(\delta, \varepsilon)$ contains the monomial $n \theta_{k^{*}}^{2} \delta^{2 k^{*}}$. We have $P(\delta, \varepsilon)=n \theta_{0}^{2}$ for $\theta=\left(0, \theta_{0}, 0, \ldots, 0\right)^{\top}$. Therefore $P(\delta, \varepsilon)$ is a nonzero polynomial. Then $\theta^{\top} \Psi\left(\kappa_{1}\right) \theta=\mathrm{E} P(\delta, \varepsilon)>0$. Here we used the explicit structure of the matrix $\Psi\left(\kappa_{2}\right)$ (a certain entry of the matrix equals $n$, see (11)) and nondegeneracy of the matrix $\Omega_{0}$.

Proof of Theorem 2.1. The matrix $\Psi(0)$, as a sum of nonnegative definite matrices, is nonnegative definite. The first entries of the matrix $\Psi(\kappa)$ are

$$
\Psi(\kappa)=\left(\begin{array}{ccc}
\sum_{i=1}^{n} y_{i}^{2}-n \kappa \sigma_{\varepsilon 0}^{2} & \sum_{i=1}^{n} y_{i} & \ldots \\
\sum_{i=1}^{n} y_{i} & n & \ldots \\
\ldots \ldots \ldots \ldots \ldots \ldots \ldots \ldots & \ldots \ldots \ldots
\end{array}\right) .
$$

The above entries of the matrix $\Psi(\kappa)$ are used to check that this matrix is either positive or negative definite. This implies that $\Psi(\kappa) \not \leq 0$ (that is, $\lambda_{\max }(\Psi(\kappa)) \geq n>0$ ) for all $\kappa \geq 0$ and that the matrix $\Psi(\kappa)$ is not definite for $\kappa>\sigma_{\varepsilon 0}^{-2} n^{-1} \sum_{i=1}^{n} y_{i}^{2}$. The entries of the matrix $\Psi(\kappa)$ are polynomials of $\kappa$. Thus a solution exists on the interval $\left[0, \sigma_{\varepsilon 0}^{-2} n^{-1} \sum_{i=1}^{n} y_{i}^{2}\right]$, since the function $\kappa \mapsto \lambda_{\min }(\Psi(\kappa))$ is continuous. The uniqueness of the solution as well as all other statements of the theorem follows from Lemma 4.2

Corollary 4.3. The symmetric matrix $\Psi_{0}(\kappa)$ is positive definite for $0 \leq \kappa<\hat{\kappa}$, is not definite for $\kappa>\hat{\kappa}$, and is degenerate for $\kappa=\hat{\kappa}$. The matrices $\Psi_{j}(\kappa), j=0, \ldots, k$, are positive definite for $0 \leq \kappa \leq \hat{\kappa}$.

4.4. The estimator of the parameter $\bar{\beta}$. Since $\Psi_{0}(\hat{\kappa})$ is a degenerate matrix, there exists a nonzero measurable solution of the equation

$$
\Psi_{0}(\hat{\kappa}) \hat{\theta}=0
$$

denoted by $\hat{\theta}=\left(\hat{\theta}_{-1}, \hat{\theta}_{0}, \ldots, \hat{\theta}_{k}\right)^{\top}$. Equations (21) and (12) are related to each other as follows:

$$
\left(\begin{array}{c}
-1 \\
\hat{\beta}
\end{array}\right)=-\frac{1}{\hat{\theta}_{-1}} K(\hat{\kappa})^{-\top} \hat{\theta}
$$

if $\hat{\kappa}$ is fixed. If $\hat{\theta}$ is a solution of (12) and $\hat{\theta}_{-1} \neq 0$, then (13) determines a solution of (2). Similarly if $\hat{\beta}$ is a solution of (2), then the vector $\hat{\theta}=K(\hat{\kappa})^{\top}\left(\begin{array}{c}-1 \\ \hat{\beta}\end{array}\right)$ is a solution of equation (12). We assume that either (13) holds or $\hat{\theta}_{-1}=0$.

Put

$$
\tilde{\theta}=\left(\tilde{\theta}_{-1}, \tilde{\theta}_{0}, \ldots, \tilde{\theta}_{k}\right)^{\top}=K(\bar{\kappa})^{\top}\left(\begin{array}{c}
-1 \\
\bar{\beta}
\end{array}\right) .
$$

Assumption (iii) of Theorem 3.1 implies that $\tilde{\theta}_{k}=\bar{\beta}_{k} \neq 0$. Note that $\tilde{\theta}_{-1}=-1$ and moreover

$$
\bar{\Psi}_{0} \tilde{\theta}=0 .
$$




\section{The COnsistency of the estimator}

Recall that conditions (i), (ii), and (iii) were introduced in Theorem 3.1

Lemma 5.1. If condition (ii) holds, then

$$
\frac{1}{n}\|\Psi(\bar{\kappa})-\bar{\Psi}\| \stackrel{\mathrm{P} 1}{\longrightarrow} 0, \quad n \rightarrow \infty .
$$

Proof. The entry $\theta_{l}^{\top} \psi(x, y, \bar{\kappa}) \theta_{r}$ of the matrix $\psi(x, y, \kappa)$ is a polynomial in $x$ and $y$. Every monomial of this polynomial divides either $y^{2}$, or $x^{k} y$, or $x^{2 k}$. Here $\theta_{l}$ and $\theta_{r}$ are basis vectors of the space $\mathbb{R}^{k+2}$. The coefficients of the polynomial depend on $\bar{\kappa} \Omega_{0}, \theta_{l}$, and $\theta_{r}$. The variance of an entry of the matrix, $\mathrm{D}\left[\theta_{l}^{\top} \psi(x, y, \bar{\kappa}) \theta_{r}\right]$, is a polynomial in $\xi$ and $\eta$ if $\left(\begin{array}{l}x \\ y\end{array}\right) \sim N\left(\left(\begin{array}{l}\xi \\ \eta\end{array}\right), \bar{\kappa} \Omega_{0}\right)$. Every monomial of the latter polynomial divides either $\xi^{2 k-2} \eta^{2}$, or $\xi^{3 k-2} \eta$, or $\xi^{4 k-2}$. The variance $\mathrm{D}\left[\theta_{l}^{\top} \psi(x, y, \bar{\kappa}) \theta_{r}\right]$ is a polynomial in the argument $\xi$ whose degree does not exceed $4 k-2$ if $\left(\begin{array}{l}x \\ y\end{array}\right) \sim N\left(\left(\begin{array}{c}\xi \\ \sum_{j=0}^{k} \beta_{j} \xi^{j}\end{array}\right), \bar{k} \Omega_{0}\right)$.

Therefore condition (ii) implies that

$$
\sum_{n=1}^{\infty} \frac{\mathrm{D}\left[\theta_{l}^{\top} \psi\left(x_{i}, y_{i}, \bar{\kappa}\right) \theta_{r}\right]}{n^{2}}<\infty
$$

According to the strong law of large numbers,

$$
\frac{1}{n}(\Psi(\bar{\kappa})-\bar{\Psi}) \stackrel{P 1}{\longrightarrow} 0 .
$$

Corollary 5.2. Assumption (ii) implies that

$$
\frac{1}{n}\left\|\Psi_{j}(\bar{\kappa})-\bar{\Psi}_{j}\right\| \stackrel{\mathrm{P} 1}{\longrightarrow} 0, \quad n \rightarrow \infty,
$$

for $j=0, \ldots, k$.

Lemma 5.3. Let conditions (i) and (iii) hold. If $P(\xi)$ is a polynomial whose degree does not exceed $2 k-2$ and $\theta_{n} \rightarrow \tilde{\theta}$ as $n \rightarrow \infty$, then there exists a constant $C_{2}$ such that

$$
\sum_{i=1}^{n}\left|P\left(\xi_{i}\right)\right| \leq C_{2} \theta_{n}^{\top} \bar{\Psi}_{1} \theta_{n}
$$

for sufficiently large $n$.

Proof. It follows from condition (iii) that $\tilde{\theta}^{\top} \tilde{\psi}_{1}(\xi) \tilde{\theta}$ is a polynomial of degree $2 k-2$. Thus the limit

$$
\lim _{\substack{\xi \rightarrow \infty \\ \theta \rightarrow \tilde{\theta}}} \frac{P(\xi)}{\theta^{\top} \tilde{\psi}_{1}(\xi) \theta}
$$

is finite. Therefore there are constants $C, E$, and $N$ such that

$$
\frac{|P(\xi)|}{\theta_{n}^{\top} \tilde{\psi}_{1}(\xi) \theta_{n}}<C, \quad|\xi| \geq E, n \geq N .
$$

One can check that $D_{y} \bar{\Psi}_{0} D_{y}^{\top}=\left(\begin{array}{cc}n & 0_{1 \times(k+1)} \\ 0_{(k+1) \times 1} & 0_{(k+1) \times(k+1)}\end{array}\right)$. Hence

$$
\theta^{\top} \bar{\Psi}_{1} \theta=\theta^{\top} R\left(\bar{\Psi}_{0}\right) \theta \geq \sigma_{\varepsilon 0}^{2} \theta^{\top} D_{y} \bar{\Psi}_{0} D_{y} \theta=\sigma_{\varepsilon 0}^{2} n \theta_{-1}^{2},
$$

where $\theta_{-1}$ is the first coordinate of the vector $\theta$. Taking into account the equality $\tilde{\theta}_{-1}=-1$, we obtain $\theta_{n}^{\top} \bar{\Psi}_{1} \theta_{n}=n / O(1)$ as $n \rightarrow \infty$. 
It is clear that

$$
\sum_{\substack{1 \leq i \leq n \\\left|\xi_{i}\right|<E}}\left|P\left(\xi_{i}\right)\right|=O(n), \quad n \rightarrow \infty,
$$

and $\theta_{n}^{\top} \tilde{\psi}_{1}\left(\xi_{i}\right) \theta_{n} \geq 0$. This means that

$$
\begin{aligned}
\frac{\sum_{i=1}^{n}\left|P\left(\xi_{i}\right)\right|}{\theta_{n}^{\top} \bar{\Psi}_{1} \theta_{n}} & =\frac{\sum_{\substack{1 \leq i \leq n \\
\left|\xi_{i}\right| \geq E}}\left|P\left(\xi_{i}\right)\right|}{\sum_{i=1}^{n} \theta_{n}^{\top} \tilde{\psi}_{1}\left(\xi_{i}\right) \theta_{n}}+\frac{\sum_{\substack{1 \leq i \leq n \\
\left|\xi_{i}\right|<E}}\left|P\left(\xi_{i}\right)\right|}{\theta_{n} \bar{\Psi}_{1} \theta_{n}} \leq C+\frac{\sum_{\substack{1 \leq i \leq n \\
\left|\xi_{i}\right|<E}}\left|P\left(\xi_{i}\right)\right|}{\theta_{n}^{\top} \bar{\Psi}_{1} \theta_{n}} \\
& =O(1), \quad n \rightarrow \infty .
\end{aligned}
$$

Corollary 5.4. Conditions (i) and (iii) imply that

$$
\left\|\bar{\Psi}_{j}\right\|=\tilde{\theta}^{\top} \bar{\Psi}_{1} \tilde{\theta} O(1), \quad n \rightarrow \infty
$$

for $j=1,2$.

Lemma 5.5. Conditions (i), (ii), and (iii) imply that

$$
(\hat{\kappa}-\bar{\kappa})^{+}=\frac{\tilde{\theta}^{\top} \Psi_{0}(\bar{\kappa}) \tilde{\theta}}{\tilde{\theta}^{\top} \bar{\Psi}_{1} \tilde{\theta}} O(1), \quad n \rightarrow \infty,
$$

almost surely. Here $x^{+}=\max \{x, 0\}$.

Proof. Consider the polynomial

$$
P(\lambda):=\tilde{\theta}^{\top} \Psi_{0}(\bar{\kappa}) \tilde{\theta}-\lambda \tilde{\theta}^{\top} \Psi_{1}(\bar{\kappa}) \tilde{\theta}+\frac{1}{2} \lambda^{2} \tilde{\theta}^{\top} \Psi_{2}(\bar{\kappa}) \tilde{\theta} .
$$

Using Corollary 4.3 we obtain the inequalities $\tilde{\theta}^{\top} \Psi_{0}(\bar{\kappa}) \tilde{\theta}>0, \tilde{\theta}^{\top} \Psi_{1}(\bar{\kappa}) \tilde{\theta} \geq 0$, and $\tilde{\theta}^{\top} \Psi_{2}(\bar{\kappa}) \tilde{\theta} \geq 0$ for $\hat{\kappa}>\bar{\kappa}$.

If $k \geq 2$, then $\tilde{\theta}^{\top} \Psi_{2}(\bar{\kappa}) \tilde{\theta} \neq 0$ almost surely.

The discriminant (the squared coefficient of $\lambda$ ) of the polynomial $P(\lambda)$,

$$
D:=\left(\tilde{\theta}^{\top} \Psi_{1}(\bar{\kappa}) \tilde{\theta}\right)^{2}-2 \tilde{\theta}^{\top} \Psi_{0}(\bar{\kappa}) \tilde{\theta} \tilde{\theta}^{\top} \Psi_{2}(\bar{\kappa}) \tilde{\theta},
$$

is positive by Corollary [5.2, Lemma [5.3, and in view of (15).

If $\hat{\kappa}>\bar{\kappa}$ and $D>0$, then the polynomial $P(\lambda)$ has a unique positive root provided $\tilde{\theta}^{\top} \Psi_{2}(\bar{\kappa}) \tilde{\theta} \neq 0$ and $P(\lambda)$ has two different positive roots if $\tilde{\theta}^{\top} \Psi_{2}(\bar{\kappa}) \tilde{\theta}=0$. The unique (if $\tilde{\theta}^{\top} \Psi_{2}(\bar{\kappa}) \tilde{\theta} \neq 0$ ) or minimal (if $\tilde{\theta}^{\top} \Psi_{2}(\bar{\kappa}) \tilde{\theta}=0$ ) root is equal to

$$
\frac{2 \tilde{\theta}^{\top} \Psi_{0} \tilde{\theta}}{\tilde{\theta}^{\top} \Psi_{1} \tilde{\theta}+\sqrt{D}} .
$$

Implying the Taylor formula for $\Psi_{0}(\kappa)$ (use (9) to evaluate derivatives), we prove that, for all $\kappa>\bar{\kappa}$, there exists a number $\kappa_{1}$ such that $\bar{\kappa}<\kappa_{1}<\kappa$ and

$$
\tilde{\theta}^{\top} \Psi_{0}(\kappa) \tilde{\theta}=P(\kappa-\bar{\kappa})-\frac{(\kappa-\bar{\kappa})^{3}}{6} \tilde{\theta}^{\top} \Psi_{3}\left(\kappa_{1}\right) \tilde{\theta} .
$$

Hence

$$
P(\lambda) \geq 0, \quad 0<\lambda \leq \hat{\kappa}-\bar{\kappa},
$$

by Corollary 4.3 if $\hat{\kappa}>\bar{\kappa}$.

Thus

$$
\hat{\kappa}-\bar{\kappa}<\frac{2 \tilde{\theta}^{\top} \Psi_{0} \tilde{\theta}}{\tilde{\theta}^{\top} \Psi_{1} \tilde{\theta}+\sqrt{D}}
$$

if the random events $\hat{\kappa}>\bar{\kappa}$ occur and $D>0$. This inequality together with Corollary 5.2 and Lemma 5.3 completes the proof. 
Lemma 5.6. Let $\left\{v_{n}, n=0,1,2, \ldots\right\} \subset \mathbb{R}^{m} \backslash\{0\}$ be a sequence of vectors such that $v_{0}^{(1)}=1$, where $v_{n}^{(1)}$ denotes the first coordinate of the vector $v_{n}$. If $\sin \angle\left(v_{n}, v_{0}\right) \rightarrow 0$ as $n \rightarrow \infty$, then

(1) $v_{n}^{(1)} \neq 0$ for sufficiently large $n$;

(2) $\left\|v_{n} / v_{n}^{(1)}-v_{0}\right\|=O\left(\sin \angle\left(v_{n}, v_{0}\right)\right)$ as $n \rightarrow \infty$.

Proof. Applying the upper bound for the difference of normed vectors (see 7 , formula (C.2)]) we get

$$
\min \left\{\left\|\frac{v_{n}}{\left\|v_{n}\right\|}-\frac{v_{0}}{\left\|v_{0}\right\|}\right\|,\left\|\frac{v_{n}}{\left\|v_{n}\right\|}+\frac{v_{0}}{\left\|v_{0}\right\|}\right\|\right\} \leq \sqrt{2} \sin \angle\left(v_{n}, v_{0}\right) \rightarrow 0, \quad n \rightarrow \infty .
$$

Thus $\left|v_{n}^{(1)}\right| /\left\|v_{n}\right\| \rightarrow 1 /\left\|v_{0}\right\| \neq 0$ as $n \rightarrow \infty$. This proves the first statement of the lemma.

The function $f(v)=v / v^{(1)}$ is differentiable on the set $\left\{v \in \mathbb{R}^{m} \mid v^{(1)} \neq 0\right\}$,

$$
f\left(\frac{v_{0}}{\left\|v_{0}\right\|}\right)=f\left(-\frac{v_{0}}{\left\|v_{0}\right\|}\right)=v_{0}, \quad f\left(\frac{v_{n}}{\left\|v_{n}\right\|}\right)=\frac{1}{v_{n}^{(1)}} v_{n},
$$

whence the second statement of the lemma follows by (17).

Proof of Theorem 3.1. 1. First we show that

$$
\frac{\hat{\theta}^{\top} \bar{\Psi}_{0} \hat{\theta}}{n\|\hat{\theta}\|^{2}} \stackrel{\mathrm{P} 1}{\longrightarrow} 0, \quad n \rightarrow \infty .
$$

Consider the random event $\hat{\kappa}>\bar{\kappa}$. By Taylor's formula, there exists a number $\kappa_{1}$ such that $\bar{\kappa}<\kappa_{1}<\hat{\kappa}$ and

$$
\hat{\theta}^{\top} \Psi_{0}(\hat{\kappa}) \hat{\theta}=\hat{\theta}^{\top} \Psi_{0}(\bar{\kappa}) \hat{\theta}-(\hat{\kappa}-\bar{\kappa}) \hat{\theta}^{\top} \Psi_{1}(\bar{\kappa}) \hat{\theta}+\frac{(\hat{\kappa}-\bar{\kappa})^{2}}{2} \hat{\theta}^{\top} \Psi_{2}\left(\kappa_{1}\right) \hat{\theta} .
$$

If $\hat{\kappa}>\bar{\kappa}$, then

$$
0 \leq \hat{\theta}^{\top} \Psi_{0}(\bar{\kappa}) \hat{\theta} \leq(\hat{\kappa}-\bar{\kappa}) \hat{\theta}^{\top} \Psi_{1}(\bar{\kappa}) \hat{\theta}
$$

by equality (12) and Corollary 4.3 .

Now we apply equality (12) together with Corollaries 5.2 and 5.4 and Lemma 5.5 to prove the relation

$$
(\hat{\kappa}-\bar{\kappa})^{+} \frac{\hat{\theta}^{\top} \Psi_{1}(\bar{\kappa}) \hat{\theta}}{n\|\hat{\theta}\|^{2}} \stackrel{\mathrm{P} 1}{\longrightarrow} 0, \quad n \rightarrow \infty .
$$

Thus inequalities (19) imply that

$$
\frac{\hat{\theta}^{\top} \bar{\Psi}_{0} \hat{\theta}}{n\|\hat{\theta}\|^{2}} \mathbf{1}\{\hat{\kappa}>\bar{\kappa}\} \stackrel{\mathrm{P} 1}{\longrightarrow} 0, \quad n \rightarrow \infty .
$$

Consider the random event $0 \leq \hat{\kappa} \leq \bar{\kappa}$. If all the coefficients of a sequence of polynomials converge, then the polynomials converge uniformly on every bounded interval. Thus equality (9) and Corollary 5.2 imply

$$
\frac{1}{n}\left(\Psi_{0}(\hat{\kappa})-\sum_{j=0}^{k} \frac{(\bar{\kappa}-\hat{\kappa})^{j}}{j !} \bar{\Psi}_{j}\right) \mathbf{1}\{\hat{\kappa} \leq \bar{\kappa}\} \stackrel{\mathrm{P} 1}{\longrightarrow} 0, \quad n \rightarrow \infty .
$$

If $\hat{\kappa} \leq \bar{\kappa}$, then

$$
\sum_{j=1}^{k} \frac{(\bar{\kappa}-\hat{\kappa})^{j}}{j !} \bar{\Psi}_{j} \geq 0
$$

by Corollary 4.3. 
We also have $\hat{\theta}^{\top} \Psi_{0}(\hat{\kappa}) \hat{\theta}=0$ by formula (12). These results yield

$$
\varlimsup_{n \rightarrow \infty}\left(\frac{\hat{\theta}^{\top} \bar{\Psi}_{0} \hat{\theta}}{n\|\hat{\theta}\|^{2}} \mathbf{1}\{\hat{\kappa} \leq \bar{\kappa}\}\right) \leq 0
$$

almost surely. Since the matrix $\bar{\Psi}_{0}$ is positive definite, convergence (18) is proved.

2. The consistency of $\hat{\theta}$. If the inequality in assumption (i) holds, then

$$
\lambda_{2}(\bar{\Psi})>n / C_{1},
$$

whence

$$
\lambda_{2}\left(\bar{\Psi}_{0}\right)>\frac{n}{C_{1}\|K(\bar{\kappa})\|^{2}},
$$

where $\lambda_{2}$ is the second minimal eigenvalue. Since $\tilde{\theta}$ is the eigenvector of $\bar{\Psi}_{0}$ corresponding to the minimal eigenvalue 0 , we obtain

$$
\lambda_{2}\left(\bar{\Psi}_{0}\right) \sin ^{2} \angle(\hat{\theta}, \tilde{\theta}) \leq \frac{\hat{\theta} \bar{\Psi}_{0} \hat{\theta}}{\|\hat{\theta}\|^{2}} .
$$

Thus (18) implies that

$$
\sin \angle(\hat{\theta}, \tilde{\theta}) \stackrel{\mathrm{P} 1}{\longrightarrow} 0, \quad n \rightarrow \infty .
$$

By Lemma 5.6 .

$$
\mathrm{P}\left(\exists n_{0} \forall n \geq n_{0}: \hat{\theta}_{-1} \neq 0\right)=1
$$

and

$$
-\frac{1}{\hat{\theta}_{-1}} \hat{\theta} \stackrel{\mathrm{P} 1}{\longrightarrow} \tilde{\theta}, \quad n \rightarrow \infty .
$$

3. The consistency of $\hat{\kappa}$. Similarly to the proof of (22) but using the inequality

$$
\bar{\Psi}_{0}+\sum_{j=2}^{k} \frac{(\bar{\kappa}-\hat{\kappa})^{j}}{j !} \bar{\Psi}_{j} \geq 0, \quad \hat{\kappa} \leq \bar{\kappa},
$$

instead of (21) we prove the convergence

$$
\frac{\hat{\theta}^{\top} \bar{\Psi}_{1} \hat{\theta}}{n\|\hat{\theta}\|^{2}}(\bar{\kappa}-\hat{\kappa})^{+} \stackrel{P 1}{\longrightarrow} 0 .
$$

Then $\|\hat{\theta}\|=-\hat{\theta}_{-1} O(1)$ almost surely in view of convergence (24), whence $(\bar{\kappa}-\hat{\kappa})^{+} \stackrel{\mathrm{P} 1}{\longrightarrow} 0$ by Lemma 5.3 .

The convergence $(\hat{\kappa}-\bar{\kappa})^{+} \stackrel{\mathrm{P} 1}{\longrightarrow} 0$ follows from equality (15), Corollary [5.2, and Lemmas 5.3 and 5.5 .

4. The consistency of $\hat{\beta}$. The function $f(\theta, \kappa):=K^{-\top}(\kappa) \theta$ is continuous and such that

$$
f(\tilde{\theta}, \bar{\kappa})=\left(\begin{array}{c}
-1 \\
\bar{\beta}
\end{array}\right), \quad f\left(-\frac{1}{\hat{\theta}_{-1}} \hat{\theta}, \hat{\kappa}\right)=\left(\begin{array}{c}
-1 \\
\hat{\beta}
\end{array}\right)
$$

see (13) and (14). Now the convergence $\hat{\beta} \stackrel{\mathrm{P} 1}{\longrightarrow} \bar{\beta}$ follows from (24) and $\hat{\kappa} \stackrel{\mathrm{P} 1}{\longrightarrow} \bar{\kappa}$. The function $f(\theta, \kappa)$ is differentiable at the point $(\tilde{\theta}, \bar{\kappa})$. Relation (3) follows from estimates for the rate of convergence of $\hat{\kappa}$ and $-\hat{\theta} / \hat{\theta}_{-1}$. 
Proof of Theorem 3.2, 1. By definition of $\Psi_{j}(\kappa)$ and $\bar{\Psi}_{j}$, we have

$$
\left\|\Psi_{j}(\bar{\kappa})-\bar{\Psi}_{j}\right\|=O(\|\Psi(\bar{\kappa})-\bar{\Psi}\|) .
$$

Thus equality (9) and the convergence $\hat{\kappa} \stackrel{\mathrm{P} 1}{\longrightarrow} \bar{\kappa}$ imply that

$$
\Psi_{0}(\hat{\kappa})-\sum_{j=0}^{k} \frac{(\bar{\kappa}-\hat{\kappa})^{j}}{j !} \bar{\Psi}_{j}=O(\|\Psi(\bar{\kappa})-\bar{\Psi}\|), \quad n \rightarrow \infty,
$$

almost surely.

2. Rate of convergence of $\hat{\kappa}$. We prove that relations (4) and

$$
(\hat{\kappa}-\bar{\kappa})\left\|\bar{\Psi}_{j}\right\|=O(\|\Psi(\bar{\kappa})-\bar{\Psi}\|), \quad n \rightarrow \infty, j \geq 1,
$$

hold almost surely.

Since $\tilde{\theta}^{\top} \Psi_{0}(\bar{\kappa}) \tilde{\theta} \leq\left\|\Psi_{0}(\bar{\kappa})-\bar{\Psi}\right\|$, we have

$$
(\hat{\kappa}-\bar{\kappa})^{+}=\frac{O(\|\Psi(\bar{\kappa})-\bar{\Psi}\|)}{\tilde{\theta}^{\top} \bar{\Psi}_{1} \tilde{\theta}}, \quad n \rightarrow \infty,
$$

almost surely by Lemma 5.5 and inequalities (5). Thus Lemma 5.3 together with Corollary 5.4 implies that

$$
n(\hat{\kappa}-\bar{\kappa})^{+}=O(\|\Psi(\bar{\kappa})-\bar{\Psi}\|), \quad\left\|\bar{\Psi}_{j}\right\|(\hat{\kappa}-\bar{\kappa})^{+}=O(\|\Psi(\bar{\kappa})-\bar{\Psi}\|)
$$

almost surely for all $j \geq 1$ as $n \rightarrow \infty$.

Using (12), (25), and (26) we get

$$
\frac{\hat{\theta}^{\top} \bar{\Psi}_{1} \hat{\theta}}{\|\hat{\theta}\|^{2}}(\bar{\kappa}-\hat{\kappa})^{+}=O(\|\Psi(\bar{\kappa})-\bar{\Psi}\|) .
$$

Thus

$$
n(\bar{\kappa}-\hat{\kappa})^{+}=O(\|\Psi(\bar{\kappa})-\bar{\Psi}\|), \quad\left\|\bar{\Psi}_{j}\right\|(\bar{\kappa}-\hat{\kappa})^{+}=O(\|\Psi(\bar{\kappa})-\bar{\Psi}\|)
$$

almost surely for all $j \geq 1$ as $n \rightarrow \infty$ in view of Lemma 5.3 and the convergence

$$
\frac{\hat{\theta}}{\|\hat{\theta}\|} \stackrel{\mathrm{P} 1}{\longrightarrow} \frac{\tilde{\theta}}{\|\tilde{\theta}\|} .
$$

Note that $\|\tilde{\theta}\| \geq 1$.

Relations (4) and (27) are proved.

3. Relations (26), (27), and $\hat{\kappa} \stackrel{\mathrm{P} 1}{\longrightarrow} \bar{\kappa}$ imply that

$$
\Psi_{0}(\hat{\kappa})-\bar{\Psi}_{0}=O\left(n^{-1}\left\|\Psi_{0}(\kappa)-\bar{\Psi}\right\|\right), \quad n \rightarrow \infty,
$$

almost surely. Now we obtain from (12) that $\Psi_{0}(\hat{\theta}) /\|\hat{\theta}\|=O\left(n^{-1}\left\|\Psi_{0}(\kappa)-\bar{\Psi}\right\|\right)$ almost surely as $n \rightarrow \infty$.

4. Rate of convergence of $-\hat{\theta} / \hat{\theta}_{-1}$. Recall that $\hat{\theta}$ is the eigenvector of the matrix $\Psi_{0}$ corresponding to the minimal eigenvalue 0 . According to the perturbation theorem for invariant subspaces of Hermitian matrices ([9, Theorem V.3.4])

$$
\sin \angle(\hat{\theta}, \tilde{\theta}) \leq \frac{\left\|\Psi_{0}(\hat{\theta})\right\|}{\|\hat{\theta}\| \lambda_{2}\left(\bar{\Psi}_{0}\right)}=O\left(n^{-1}\left\|\Psi_{0}(\kappa)-\bar{\Psi}\right\|\right), \quad n \rightarrow \infty,
$$

almost surely. Here we used inequality (23). By Lemma 5.6,

$$
\left\|-\frac{1}{\hat{\theta}_{-1}} \hat{\theta}-\tilde{\theta}\right\|=O\left(n^{-1}\left\|\Psi_{0}(\kappa)-\bar{\Psi}\right\|\right), \quad n \rightarrow \infty,
$$

almost surely. 
5. Rate of convergence of $\hat{\beta}$. The function $f(\theta, \kappa)$ defined in step 4 of the proof of Theorem 3.1 is differentiable. Thus relation (3) follows from the estimates of the rate of convergence for $\hat{\kappa}$ and $-\hat{\theta} / \hat{\theta}_{-1}$.

\section{ImPROVEMENTS OF THE CONDITIONS}

6.1. Condition $\Omega_{0}>0$ fails. Consider the case where the matrix $\Omega_{0}$ is degenerate.

6.1.1. Case of $\sigma_{\delta 0}^{2}=0$. This case corresponds to a polynomial regression without measurement errors in the independent variable. Our estimator coincides with the usual least squares estimator in this case. The estimator is consistent if (i) holds and $\sum_{n=1}^{\infty} \xi_{n}^{2 k}<\infty$. Note that the latter condition is weaker than (ii).

6.1.2. Case of $\sigma_{\varepsilon 0}^{2}=0$. We assumed that $\Omega_{0}$ is nondegenerate in Lemmas 4.2 and 5.3 and Theorem 2.1 .

Lemma 4.2 does not hold if the random event $y_{1}=y_{2}=\cdots=y_{n}$ occurs. Then the equation $\lambda_{\min }(\Psi(\kappa))=0$ has several solutions. Condition (i) implies that this random event occurs with probability 0 if $n$ is sufficiently large.

The equation $\lambda_{\min }(\Psi(\kappa))=0$ has at least one solution in the interval

$$
\left[0, \frac{1}{n \sigma_{\delta 0}^{2}} \sum_{i=1}^{n} x_{i}^{2}\right) .
$$

The proof of Lemma 5.3 becomes more involved in this case, but its main result still holds.

The main theorems 3.1 and 3.2 remain true in the case of $\sigma_{\varepsilon 0}^{2}=0$.

6.2. Dependence between measurement errors and the echo. Suppose the covariance matrix of the measurement errors $\Omega_{0}=\left(\begin{array}{cc}\sigma_{\delta 0}^{2} & \sigma_{\delta \varepsilon 0} \\ \sigma_{\delta \varepsilon 0} & \sigma_{\varepsilon 0}^{2}\end{array}\right)>0$ is not necessarily diagonal. Recall that $\left(\delta_{i}, \varepsilon_{i}\right)^{\top} \sim N\left(0, \Omega_{0}\right), i=1,2, \ldots$. In this case, we assume that the vectors $\left(\delta_{i}, \varepsilon_{i}\right), i=1,2, \ldots$, are jointly independent but allow $\delta_{i}$ and $\varepsilon_{i}$ to be dependent. The estimator is defined as in Section 2. (The explicit form of the matrix $\psi(x, y, \kappa)$ becomes more complicated. Moreover relations (11) and $\psi_{-1, i}=\psi_{i,-1}=y t_{i}(x)$ do not hold.) Theorems 3.1 and 3.2 remain true. The proof uses the property that the estimator is equivariant.

6.3. Case of $\beta_{k}=0$ (assumption (iii) fails). We denote by $d$ the degree of the polynomial

$$
\sum_{j=0}^{k} \bar{\beta}_{k} \xi^{k}
$$

which describes the dependence of $\eta_{i}$ on $\xi_{i}$. We set $d=0$ if $\bar{\beta}=0$.

We provide below an analogue of Lemma 5.3 for the case where condition (iii) fails.

Lemma 6.1. Suppose condition $(i)$ holds, and let $P(\xi)$ be a polynomial whose degree does not exceed $\max \{2 d-2,0\}$, and $\theta_{n} \rightarrow \tilde{\theta}$ as $n \rightarrow \infty$. Then there exists a constant $C_{2}$ such that inequality (16) holds for sufficiently large $n$.

In the course of the proof of Lemma 6.1 we show that $\tilde{\theta}^{\top} \tilde{\psi}_{1}(\xi) \tilde{\theta}$ is a nonzero polynomial of degree

$$
\max \{2 d-2,0\}
$$

Condition (iii) is not assumed in Lemma 5.5. The degree of the polynomial $\tilde{\theta}^{\top} \Psi_{1} \tilde{\theta}$ equals $\max \{2 d-1,0\}$. Lemma 6.1 can be used instead of Lemma 5.3 . 
Note however that the proof of the consistency of the estimator $\hat{\beta}$ does not work without condition (iii), since convergence (20) is not justified. Nevertheless $\hat{\kappa}$ is a consistent estimator. One should use the relation $\left(-\hat{\theta} / \hat{\theta}_{-1}-\tilde{\theta}\right) \mathbf{1}\{\hat{\kappa} \leq \bar{\kappa}\} \stackrel{\mathrm{P} 1}{\longrightarrow} 0$ instead of (24) in the proof.

6.4. The distribution of $\varepsilon$ is not Gaussian. We drop the assumption that

$$
\left(\begin{array}{l}
\delta_{i} \\
\varepsilon_{i}
\end{array}\right) \sim N\left(0, \bar{\kappa} \Omega_{0}\right)
$$

Instead we assume that $\delta_{i} \sim N\left(0, \bar{\kappa} \sigma_{\delta 02}^{2}\right), \mathrm{E} \varepsilon_{i}=0, \mathrm{D} \varepsilon_{i}=\bar{\kappa} \sigma_{\varepsilon 0}^{2}$, and $\delta_{i}$ and $\varepsilon_{i}$ are independent for all $i \geq 1$. Furthermore, we assume that the law of large numbers

$$
\frac{1}{n} \sum_{i=1}^{n} \varepsilon_{i}^{2} \stackrel{\mathrm{P} 1}{\longrightarrow} \bar{\kappa} \sigma_{\varepsilon 0}^{2}
$$

holds almost surely.

Lemma 5.1 holds under these assumptions; thus so do Theorems 3.1 and 3.2 .

\section{The StruCtural MOdel}

We assumed above that the $\xi_{i}$ are nonrandom. Now we assume that $\left\{\xi_{i}, i=1,2, \ldots\right\}$ are independent identically distributed random variables. The echo is $\eta_{i}=\sum_{j=1}^{n} \bar{\beta}_{j} \xi_{i}^{j}$, $i=1,2, \ldots$. We observe the sample $\left\{\left(x_{i}, y_{i}\right), i=1, \ldots, n\right\}$, where

$$
\left(x_{i}, y_{i}\right)=\left(\xi_{i}, \eta_{i}\right)+\left(\delta_{i}, \varepsilon_{i}\right)
$$

and $\left(\delta_{i}, \varepsilon_{i}\right) \sim N\left(0, \bar{\kappa} \Omega_{0}\right)$ is the measurement error. The random variables $\xi_{1}, \xi_{2}, \ldots$, $\delta_{1}, \delta_{2}, \ldots, \varepsilon_{1}, \varepsilon_{2}, \ldots$ are jointly independent. The covariance matrix of the error $\bar{\kappa} \Omega_{0}$ is known up to the scalar factor $\bar{\kappa}$. Moreover $\Omega_{0}=\operatorname{diag}\left(\sigma_{\delta 0}^{2}, \sigma_{\varepsilon 0}^{2}\right)$ where $\sigma_{\delta 0}>0$ and $\sigma_{\varepsilon 0}>0$.

We use the bounds given in Section 2 for the parameters $\bar{\kappa}$ and $\bar{\beta}$.

Proof of Theorem 3.3. Condition $(\mathrm{Si})$ implies that $\mathrm{P}\left(\sum_{j=0}^{m} \beta_{j} \xi_{1}^{j}=0\right)<1$ for an arbitrary $\beta \in \mathbb{R}^{k+1}, \beta \neq 0$. Thus

$$
\mathrm{E} \frac{1}{1+\xi_{1}^{2 k}}\left(\begin{array}{ccc}
1 & \ldots & \xi_{1}^{k} \\
\ldots \ldots & \ldots & \ldots \\
\xi_{1}^{k} & \ldots & \xi_{1}^{2 k}
\end{array}\right)>0
$$

According to the strong law of large numbers,

$$
\frac{1}{n} \sum_{i=1}^{n} \frac{1}{1+\xi_{i}^{2 k}}\left(\begin{array}{ccc}
1 & \ldots & \xi_{i}^{k} \\
\ldots & \ldots & \ldots \\
\xi_{i}^{k} & \ldots & \xi_{i}^{2 k}
\end{array}\right) \stackrel{\mathrm{P} 1}{\longrightarrow} \mathrm{E} \frac{1}{1+\xi_{1}^{2 k}}\left(\begin{array}{ccc}
1 & \ldots & \xi_{1}^{k} \\
\ldots & \ldots & \ldots \\
\xi_{1}^{k} & \ldots & \xi_{1}^{2 k}
\end{array}\right) .
$$

Hence

$$
\begin{aligned}
\varliminf_{n \rightarrow \infty} \lambda_{\min }\left(\frac{1}{n} \sum_{i=1}^{n}\left(\begin{array}{ccc}
1 & \ldots & \xi_{i}^{k} \\
\ldots \ldots & \ldots . & \ldots . \\
\xi_{i}^{k} & \ldots & \xi_{i}^{2 k}
\end{array}\right)\right) & \geq \lim _{n \rightarrow \infty} \lambda_{\min }\left(\frac{1}{n} \sum_{i=1}^{n} \frac{1}{1+\xi_{i}^{2 k}}\left(\begin{array}{ccc}
1 & \ldots & \xi_{i}^{k} \\
\ldots & \ldots & \ldots \\
\xi_{i}^{k} & \ldots & \xi_{i}^{2 k}
\end{array}\right)\right) \\
& =\lambda_{\min }\left(\mathrm{E} \frac{1}{1+\xi_{1}^{2 k}}\left(\begin{array}{ccc}
1 & \ldots & \xi_{1}^{k} \\
\ldots \ldots & \ldots & \ldots \\
\xi_{1}^{k} & \ldots & \xi_{1}^{2 k}
\end{array}\right)\right)>0
\end{aligned}
$$

almost surely. This implies condition (i).

Note that the matrix $\Psi(\bar{\kappa})-\bar{\Psi}$ is a sum of the terms

$$
\psi\left(\xi_{i}+\delta_{i}, \sum_{j=0}^{k} \bar{\beta}_{j} \xi_{i}^{j}+\varepsilon_{i}, \bar{\kappa}\right)-\psi\left(\xi_{i}, \sum_{j=0}^{k} \bar{\beta}_{j} \xi_{i}^{j}\right),
$$


where the index $i$ runs in the range $1, \ldots, n$. Every term of this sum is a matrix whose entries are polynomials in the variables $\xi, \varepsilon$, and $\delta$. The powers of $\xi$ in these polynomials do not exceed $2 k-1$. To obtain this result we note that every entry of the matrix $\psi(\xi, \eta, \kappa)$ is a polynomial in the arguments $\xi$ and $\eta$. Each monomial of these polynomials divides either $\eta^{2}$, or $\xi^{k} \eta$, or $\xi^{2 k}$. Moreover the expressions for the corner entries of $\psi(\xi, \eta, \kappa)$ include $\eta^{2}, \xi^{k} \eta$, and $\xi^{2 k}$. Namely

$$
\psi(\xi, \eta, \kappa)=\left(\begin{array}{ccc}
\eta^{2} & 0_{1 \times k} & \xi^{k} \eta \\
0_{k \times 1} & 0_{k \times k} & 0_{k \times 1} \\
\xi^{k} \eta & 0_{1 \times k} & \xi^{2 k}
\end{array}\right)+r_{\kappa \Omega_{0}}(\xi, \eta)
$$

where $r_{\kappa \Omega_{0}}(\xi, \eta)$ is a matrix whose entries are polynomials and every monomial of those polynomials divides either $\xi^{k-1} \eta$ or $\xi^{2 k-1}$. The terms with $\eta^{2}, \xi^{k} \eta$, and $\xi^{2 k}$ have the same coefficients in the entries of the matrix $\psi(\xi+\delta, \eta+\varepsilon, \kappa)$, that is,

$$
\psi(\xi+\delta, \eta+\varepsilon, \kappa)=\left(\begin{array}{ccc}
\eta^{2} & 0_{1 \times k} & \xi^{k} \eta \\
0_{k \times 1} & 0_{k \times k} & 0_{k \times 1} \\
\xi^{k} \eta & 0_{1 \times k} & \xi^{2 k}
\end{array}\right)+r_{\kappa \Omega_{0}}(\xi, \eta, \delta, \varepsilon)
$$

where $r_{\kappa \Omega_{0}}(\xi, \eta, \delta, \varepsilon)$ is a matrix whose entries are polynomials and every monomial of those polynomials divides either $\xi^{k-1} \eta \delta^{2 k} \varepsilon^{2}$ or $\xi^{2 k-1} \delta^{2 k} \varepsilon^{2}$. Thus

$$
\psi(\xi+\delta, \eta+\varepsilon, \bar{\kappa})-\psi(\xi, \eta)=r_{\bar{\kappa} \Omega_{0}}(\xi, \eta, \delta, \varepsilon)-r_{0_{2 \times 2}}(\xi, \eta) .
$$

Every entry of the matrix

$\psi\left(\xi+\delta, \sum_{j=0}^{k} \bar{\beta}_{j} \xi^{j}+\varepsilon, \bar{\kappa}\right)-\psi\left(\xi, \sum_{j=0}^{k} \bar{\beta}_{j} \xi^{j}\right)=r_{\bar{\kappa} \Omega_{0}}\left(\xi, \sum_{j=0}^{k} \bar{\beta}_{j} \xi^{j}, \delta, \varepsilon\right)-r_{0_{2 \times 2}}\left(\xi, \sum_{j=0}^{k} \bar{\beta}_{j} \xi^{j}\right)$

is a polynomial and each monomial of this polynomial divides $\xi^{2 k-1} \delta^{2 k} \varepsilon^{2}$.

Since all moments of $\varepsilon_{1}$ and $\delta_{1}$ exist and $\xi_{1}$ and $\left(\varepsilon_{1}, \delta_{1}\right)$ are independent, condition (Sii) implies that the expectation

$$
\mathrm{E}\left[\psi\left(x_{1}, y_{1}, \bar{\kappa}\right)-\psi\left(\xi_{1}, \eta_{1}\right)\right]=\mathrm{E}\left[\psi\left(\xi_{1}+\delta_{1}, \sum_{j=0}^{k} \bar{\beta}_{j} \xi_{1}^{j}+\varepsilon_{1}, \bar{\kappa}\right)-\psi\left(\xi_{1}, \sum_{j=0}^{k} \bar{\beta}_{j} \xi_{1}^{j}\right)\right]
$$

is finite. Since $\mathrm{E}\left[\psi\left(x_{1}, y_{1}, \bar{\kappa}\right)-\psi\left(\xi_{1}, \eta_{1}\right) \mid \xi_{1}\right]=0$, we get $\mathrm{E}\left[\psi\left(x_{1}, y_{1}, \bar{\kappa}\right)-\psi\left(\xi_{1}, \eta_{1}\right)\right]=0$.

The strong law of large numbers implies that $n^{-1}(\Psi(\bar{\kappa})-\bar{\Psi}) \stackrel{\mathrm{P} 1}{\longrightarrow} 0$ as $n \rightarrow \infty$.

Now one can repeat the reasoning of Section 5 and complete the proof.

Remark 7.1. Condition (iii) is not essential for Theorem 3.3. One can prove the consistency of the estimator $\hat{\kappa} \stackrel{\mathrm{P} 1}{\longrightarrow} \bar{\kappa}$ without assuming condition (iii); see Section 6.3. After having proved the consistency one can deduce from (Sii) that the convergence $n^{-1}(\Psi(\hat{\kappa})-\bar{\Psi}) \stackrel{\mathrm{P} 1}{\longrightarrow} 0$ holds.

\section{Concluding Remarks}

Sufficient conditions for the strong consistency of estimators of parameters of the polynomial regression with Gaussian measurement errors are found in the paper. There is good reason to believe that these conditions are too restrictive and can be weakened.

In the case of the linear regression (that is, if $k=1$ ) we consider the least squares estimators for all variables (or the orthogonal regression estimator if $\sigma_{\delta 0}^{2}=\sigma_{\varepsilon 0}^{2}$ ). Then Theorem 3.1 holds if conditions (ii) and (iii) are dropped. The author is unaware whether conditions (ii) and (iii) can be omitted if $k \geq 2$.

For the Gallo linear regression [10], Kukush et al. 11] weaken condition (i) by using the assumption on the existence of higher moments of measurement errors. 


\section{BIBLIOGRAPHY}

1. C.-L. Cheng and J. Van Ness, Statistical Regression with Measurement Error, Arnold, London, 1999. MR 1719513 (2001k:62001)

2. C.-L. Cheng and H. Schneeweiss, Polynomial regression with measurement errors, J. Roy. Statist. Soc. Ser. B 60 (1998), 189-199. MR1625632

3. C.-L. Cheng and H. Schneeweiss, On the polynomial measurement error model, Total Least Squares and Error-In-Variables Modelling (S. Van Huffel and Ph. Lemmerling, eds.), Kluwer, Dordrecht, 2002, pp. 131-143. MR.1952942

4. G. S. Repetats'ka, Inconsistency of an orthogonal regression estimator in a vector nonlinear errors-in-variables model, Teor. Imovir. Mat. Stat. 73 (2005), 146-160; English transl. in Theory Probab. Math. Statist. 73 (2006), 163-179. MR2213850 (2007g:62072)

5. Zh. Zhang, Parameter estimation techniques, Image \& Vision Computing J. 15 (1997), no. 1, $59-76$.

6. A. Kukush, I. Markovsky, and S. Van Huffel, Consistent estimation in an implicit quadratic measurement error model, Comput. Statist. Data Anal. 47 (2004), no. 1, 123-147. MR2087933 (2005h:62077)

7. S. Shklyar, A. Kukush, I. Markovsky, and S. Van Huffel, On the conic section fitting problem, J. Multivariate Anal. 98 (2007), no. 3, 588-642. MR2293016 (2008g:62164)

8. S. Shklyar, H. Schneeweiss, and A. Kukush, Quasi score is more efficient than corrected score in a polynomial measurement error model, Metrika 65 (2007), no. 3, 275-295. MR2299552

9. G. Stewart and J. Sun, Matrix Perturbation Theory, Academic Press, London, 1990. MR.1061154 (92a:65017)

10. P. Gallo, Consistency of regression estimates when some variables are subject to error, Commun. Stat. Theor. Meth. 11 (1982), no. 9, 973-983. MR655466 (83h:62106)

11. A. G. Kukush and S. Van Huffel, Consistency of elementwise-weighted total least squares estimator in a multivariate error-in-variables model $A X=B$, Metrika 59 (2004), no. 1, 75-97. MR:2043433 (2004m:62129)

Department of Probability Theory and Mathematical Statistics, Faculty for Mechanics and Mathematics, National Taras Shevchenko University, Academician Glushkov Avenue 6, KYIV 03127, UKRAINE

E-mail address: shklyar@mail.univ.kiev.ua

Received 24/FEB/2006

Translated by N. SEMENOV 